\title{
CAUCHY PROBLEM FOR SEMILINEAR WAVE EQUATION WITH TIME-DEPENDENT METRICS
}

\author{
YAVAR KIAN
}

\begin{abstract}
We establish the existence of weak solutions $u$ of the semilinear wave equation $\partial_{t}^{2} u-\operatorname{div}_{x}\left(a(t, x) \nabla_{x} u\right)=f_{k}(u)$ where $a(t, x)$ is equal to 1 outside a compact set with respect to $x$ and a non-linear term $f_{k}$ which satisfies $\left|f_{k}(u)\right| \leq C|u|^{k}$. For some non-trapping time-periodic perturbations $a(t, x)$, we obtain the long time existence of solution for small initial data.
\end{abstract}

\section{INTRODUCTION}

Consider the semilinear Cauchy problem

$$
\left\{\begin{array}{c}
u_{t t}-\operatorname{div}_{x}\left(a(t, x) \nabla_{x} u\right)-f_{k}(u)=0, \quad(t, x) \in \mathbb{R}^{n+1} \\
\left(u, u_{t}\right)(0, x)=\left(g_{1}(x), g_{2}(x)\right)=g(x), \quad x \in \mathbb{R}^{n}
\end{array}\right.
$$

where for a given $k>1$ the non-linearity $f_{k}$ is assumed to be a $C^{1}$ function on $\mathbb{R}$ satisfying $f_{k}(0)=0,\left|f_{k}^{\prime}(u)\right| \leq C|u|^{k-1}$ and the perturbation $a(t, x) \in C^{\infty}\left(\mathbb{R}^{n+1}\right)$ satisfies the conditions:

(i) $C_{0} \geq a(t, x) \geq c_{0}>0, \forall(t, x) \in \mathbb{R}^{n+1}$,

(ii) there exists $\rho>0$ such that $a(t, x)=1$ for $|x| \geq \rho$.

Denote by $\dot{H}^{1}\left(\mathbb{R}^{n}\right)$ the closure of $\mathcal{C}_{0}^{\infty}\left(\mathbb{R}^{n}\right)$ with respect to the norm

$$
\|\varphi\|_{\dot{H}^{1}}=\left(\int_{\mathbb{R}^{n}}|\nabla \varphi(x)|^{2} d x\right)^{\frac{1}{2}} .
$$

Throughout this paper we assume that $n \geq 3$ and that the initial data $g$ is in the energy space $\dot{\mathcal{H}}_{1}\left(\mathbb{R}^{n}\right)=\dot{H}^{1}\left(\mathbb{R}^{n}\right) \times L^{2}\left(\mathbb{R}^{n}\right)$. Consider the linear problem associated to $(1.1)$

$$
\left\{\begin{array}{c}
u_{t t}-\operatorname{div}_{x}\left(a(t, x) \nabla_{x} u\right)=0, \quad(t, x) \in \mathbb{R}^{n+1}, \\
\left(u, u_{t}\right)(s, x)=\left(g_{1}(x), g_{2}(x)\right)=g(x), \quad x \in \mathbb{R}^{n},
\end{array}\right.
$$

where $g \in \dot{\mathcal{H}}_{1}\left(\mathbb{R}^{n}\right)$. The solution of (1.3) is given by the propagator

$$
\mathcal{U}(t, s): \dot{\mathcal{H}}_{1}\left(\mathbb{R}^{n}\right) \ni\left(g_{1}, g_{2}\right)=g \mapsto \mathcal{U}(t, s) g=\left(u, u_{t}\right)(t, x) \in \dot{\mathcal{H}}_{1}\left(\mathbb{R}^{n}\right) .
$$

We denote by $U(t, s)$ and $V(t, s)$ the operators defined by

$$
\begin{array}{ll}
U(t, s) f=(\mathcal{U}(t, s)(f, 0))_{1}, & f \in \dot{H}^{1}\left(\mathbb{R}^{n}\right), \\
V(t, s) h=(\mathcal{U}(t, s)(0, h))_{1}, & h \in L^{2}\left(\mathbb{R}^{n}\right),
\end{array}
$$

where $\left(h_{1}, h_{2}\right)_{1}=h_{1}$. We say that $u \in \mathcal{C}\left(\left[0, T_{1}\right], \dot{H}^{1}\right)$ is a weak solution of $(1.1)$ if for all $t \in\left[0, T_{1}\right]$ we have

$$
\begin{aligned}
u(t) & =\left(\mathcal{U}(t, 0) g+\int_{0}^{t} \mathcal{U}(t, s)\left(0, f_{k}(u(s))\right) \mathrm{d} s\right)_{1} \\
& =(\mathcal{U}(t, 0) g)_{1}+\int_{0}^{t} V(t, s)\left(f_{k}(u(s)) \mathrm{d} s .\right.
\end{aligned}
$$


Let $a(t, x)=1$. Then we have the following Cauchy problem

$$
\left\{\begin{array}{c}
u_{t t}-\Delta_{x} u-f_{k}(u)=0, \quad(t, x) \in \mathbb{R}^{n+1} \\
\left(u, u_{t}\right)(0, x)=\left(g_{1}(x), g_{2}(x)\right)=g(x), \quad x \in \mathbb{R}^{n} .
\end{array}\right.
$$

The problem (1.5) has been extensively studied for $g \in \dot{\mathcal{H}}_{1}\left(\mathbb{R}^{n}\right)$. For example, the global wellposedness of the problem (1.5) has been established for the case of the sub-critical growth $1<k<$ $1+\frac{4}{n-2}$ (see [6] and [19]) or for the case of the critical growth $k=1+\frac{4}{n-2}$ (see [15] and [19]). For the case $k>1+\frac{4}{n-2}$ it is not yet clear whether there exists or not a global regular solution for the Cauchy problem (1.5) with arbitrary initial data. On the other hand, local well posedness as well as global well-posedness, with small initial data in fractional Sobolev spaces have been also studied by many authors for the problem (1.5) under minimal regularity assumptions on the initial data (see [8] and [19]).

In [18] Michael Reissig and Karen Yagdjian established Strichartz decay estimates for the solution of strictly hyperbolic equations of second order with coefficients depending only on $t$. We can apply these estimates to prove existence results for the solution of problem (1.1) when $a(t, x)=a(t)$ is independent on $x$ (see [11] and [20] for the case of the free wave equation). It seems that our paper is one of the first works where one treats non-linear wave equations with time dependent perturbations $a(t, x)$ depending on $t$ and $x$.

The goal of this paper is to find sufficient conditions for the existence of a weak solution of (1.1) when $0 \leq t \leq T_{1}$. For this purpose, we will use Strichartz estimates to study local and long time existence and uniqueness of solutions of the problem (1.1). In fact, for suitable $k$ Strichartz estimates allow us to find a fixed point of the map

$$
\mathcal{G}(u)=(\mathcal{U}(t, 0) g)_{1}+\int_{0}^{t} V(t, s) f_{k}(u(s)) \mathrm{d} s,
$$

in $\mathcal{C}\left(\left[0, T_{1}\right], \dot{H}^{1}\right)$ for some well chosen $k>1$. The fixed point of $\mathcal{G}$ is local weak solution of (1.1). In [9] we have established local homogeneous Strichartz estimates for $n \geq 3$ and $a(t, x)$ satisfying (1.2), and global homogeneous Strichartz estimates when $n \geq 3$ is odd for some non-trapping time-periodic perturbation $a(t, x)$ ( see Section 2). Recently global Strichartz estimates for even dimensions $n \geq 4$ have been obtained in [10]. One way to obtain global weak solutions is to apply global non homogeneous Strichartz estimates concerning the solution of the Cauchy problem for $u_{t t}-\operatorname{div}(a(t, x) \nabla u(x))=G(t, x)$. This leads to some difficulties and this case is not covered by our results in [9] and [10]. On the other hand, for time dependent perturbations we have no conservation laws. For these reasons we obtain only long time existence of weak solution in Section 4. In Section 2 we recall the estimates for the linear wave equation with metric $a(t, x)$. In Section 3 we obtain local existence results, while in Section 4 we deal with long time existence.

Remark 1. Let the metric $\left(a_{i j}(t, x)\right)_{1 \leq i, j \leq n}$ be such that for all $i, j=1 \cdots n$ we have

(i) there exists $\rho>0$ such that $a_{i j}(t, x)=\delta_{i j}$, for $|x| \geq \rho$, with $\delta_{i j}=0$ for $i \neq j$ and $\delta_{i i}=1$,

(ii) there exists $T>0$ such that $a_{i j}(t+T, x)=a_{i j}(t, x),(t, x) \in \mathbb{R}^{n+1}$,

(iii) $a_{i j}(t, x)=a_{j i}(t, x),(t, x) \in \mathbb{R}^{n+1}$,

(iv) there exist $C_{0}>c_{0}>0$ such that $C_{0}|\xi|^{2} \geq \sum_{i, j=1}^{n} a_{i j}(t, x) \xi_{i} \xi_{j} \geq c_{0}|\xi|^{2}, \quad(t, x) \in \mathbb{R}^{1+n}, \xi \in \mathbb{R}^{n}$. 
If we replace $a(t, x)$ in (1.1) we get the following problem

$$
\left\{\begin{array}{c}
u_{t t}-\sum_{i, j=1}^{n} \frac{\partial}{\partial x_{i}}\left(a_{i j}(t, x) \frac{\partial}{\partial x_{j}} u\right)-f_{k}(u)=0, \quad(t, x) \in \mathbb{R}^{n+1}, \\
\left(u, u_{t}\right)(s, x)=\left(f_{1}(x), f_{2}(x)\right)=f(x), \quad x \in \mathbb{R}^{n} .
\end{array}\right.
$$

Since, with the same conditions as (1.1), global Strichartz estimates are true for solutions of this equation when $f_{k}(u)=0$ (see [10]), all the results of this paper remain true for this problem.

\section{Strichartz eStimates For the Linear EQUATION}

In this section we recall some results concerning Strichartz estimates for the problem (1.3). We suppose that $a(t, x)$ satisfies the conditions (1.2). It was established in [9] that we have the following estimates.

Theorem 1. Assume $n \geq 3$ and let $a(t, x)$ be a $C^{\infty}$ function on $\mathbb{R}^{n+1}$ satisfying conditions (1.2). Let $2 \leq p, q<+\infty, \gamma>0$ be such that

$$
\frac{1}{p}=\frac{n(q-2)}{2 q}-\gamma, \quad \frac{1}{p} \leq \frac{(n-1)(q-2)}{4 q} .
$$

Then there exists $\delta>0$ such that for the solution $u(t, x)$ of (1.3) with $s=0$ we have

$$
\|u\|_{L^{p}\left([0, \delta], L^{q}\left(\mathbb{R}_{x}^{n}\right)\right)}+\|u(t)\|_{\mathcal{C}\left([0, \delta], \dot{H}^{\gamma}\left(\mathbb{R}_{x}^{n}\right)\right)}+\left\|\partial_{t}(u)(t)\right\|_{\mathcal{C}\left([0, \delta], \dot{H}^{\gamma-1}\left(\mathbb{R}_{x}^{n}\right)\right)} \leq C(p, q, \rho, n)\left\|_{g}\right\|_{\dot{\mathcal{H}}_{\gamma}} .
$$

Now, let $a(t, x)$ be $T$-periodic with respect to $t$ which means

$$
a(t+T, x)=a(t, x), \quad \forall(t, x) \in \mathbb{R}^{n+1} .
$$

Moreover, we impose two hypothesis. The first one says that the perturbation $a(t, x)$ is nontrapping. More precisely, consider the null bicharacteristics $(t(\sigma), x(\sigma), \tau(\sigma), \xi(\sigma))$ of the principal symbol $\tau^{2}-a(t, x)|\xi|^{2}$ of $\partial_{t}^{2}-\operatorname{div}\left(a \nabla_{x} u\right)$ satisfying

$$
t(0)=0,|x(0)| \leq \rho, \quad \tau^{2}(\sigma)=a(t(\sigma), x(\sigma))|\xi(\sigma)|^{2} .
$$

We introduce the following condition. that

(H1) We say that the metric $a(t, x)$ is non-trapping if for each $R>\rho$ there exists $S_{R}>0$ such

$$
|x(\sigma)|>R \text { for }|\sigma| \geq S_{R}
$$

Notice that if we have trapping metrics, there exist solutions of (1.3) whose local energy is exponentially growing (see [2]). Thus for trapping metrics it is not possible to establish global Strichartz estimates.

Let $\psi_{1}, \psi_{2} \in \mathcal{C}_{0}^{\infty}\left(\mathbb{R}^{n}\right)$. We define the cut-off resolvent associated to problem (1.3) by $R_{\psi_{1}, \psi_{2}}(\theta)=$ $\psi_{1}\left(\mathcal{U}(T, 0)-e^{-i \theta}\right)^{-1} \psi_{2}$. Consider the following assumption.

(H2) Let $\psi_{1}, \psi_{2} \in \mathcal{C}_{0}^{\infty}\left(\mathbb{R}^{n}\right)$ be such that $\psi_{i}=1$ for $|x| \leq \rho+1+3 T, i=1,2$. Then the operator $R_{\psi_{1}, \psi_{2}}(\theta)$ admits a holomorphic extension from $\{\theta \in \mathbb{C}: \operatorname{Im}(\theta) \geq A>0\}$ to $\{\theta \in \mathbb{C}: \operatorname{Im}(\theta) \geq 0\}$, for $n \geq 3$, odd, and to $\{\theta \in \mathbb{C}: \operatorname{Im}(\theta)>0\}$ for $n \geq 4$, even. Moreover, for $\mathrm{n}$ even, $R_{\psi_{1}, \psi_{2}}(\theta)$ admits a continuous extension from $\{\theta \in \mathbb{C}: \operatorname{Im}(\theta)>0\}$ to $\{\theta \in \mathbb{C}: \operatorname{Im}(\theta) \geq 0, \theta \neq 2 k \pi, \forall k \in \mathbb{Z}\}$ and we have

$$
\limsup _{\operatorname{Im}(\lambda)>0, \lambda \rightarrow 0}\left\|R_{\psi_{1}, \psi_{2}}(\lambda)\right\|<\infty .
$$

Assuming conditions (H1) and (H2), we obtained the following estimates (see [9], [10]). 
Theorem 2. Assume $n \geq 3$ and let $a(t, x)$ be a T-periodic metric satisfying (1.2) for which the conditions (H1) and (H2) are fulfilled. Let $2 \leq p, q<+\infty$ be such that

$$
p>2, \quad \frac{1}{p}=\frac{n(q-2)}{2 q}-1, \quad \frac{1}{p} \leq \frac{(n-1)(q-2)}{4 q} .
$$

Then for the solution $u(t)$ of (1.3) with $s=0$ we have for all $t>0$ the estimate

$$
\|u(t)\|_{L^{p}\left(\mathbb{R}_{t}^{+}, L^{q}\left(R_{x}^{n}\right)\right)}+\|u(t)\|_{\dot{H}^{1}\left(\mathbb{R}_{x}^{n}\right)}+\left\|\partial_{t}(u)(t)\right\|_{L^{2}\left(\mathbb{R}_{x}^{n}\right)} \leq C(p, q, \rho, T)\left(\left\|g_{1}\right\|_{\dot{H}^{1}\left(\mathbb{R}^{n}\right)}+\left\|g_{2}\right\|_{L^{2}\left(\mathbb{R}^{n}\right)}\right) .
$$

The crucial point in the proof of the global estimates $(2.4)$ is the $L^{2}$ integrability with respect to $t$ of the local energy (see [9], [10]). For this purpose we need to show that for cut-off functions $\psi_{1}, \psi_{2} \in \mathcal{C}_{0}^{\infty}\left(\mathbb{R}^{n}\right)$ such that $\psi_{i}=1$ for $|x| \leq \rho+1+3 T, i=1,2$, for $t \geq s$ we have

$$
\left\|\psi_{1} \mathcal{U}(t, s) \psi_{2}\right\|_{\mathcal{L}\left(\dot{\mathcal{H}}_{1}\left(\mathbb{R}^{n}\right)\right)} \leq C_{\psi_{1}, \psi_{2}} d(t-s)
$$

with $d(t) \in L^{1}\left(\mathbb{R}^{+}\right)$. To obtain (2.5), we use the assumption (H2). For $n \geq 3$, odd, we have an exponential decay of energy and $d(t)=e^{-\delta t}, \delta>0$. For $n \geq 4$, even, we have another decay. In particular, the estimate

$$
\left\|\psi_{1} \mathcal{U}(N T, 0) \psi_{2}\right\|_{\mathcal{L}\left(\dot{\mathcal{H}}_{1}\left(\mathbb{R}^{n}\right)\right)} \leq \frac{C_{\psi_{1}, \psi_{2}}}{(N+1) \ln ^{2}(N+e)}, \quad \forall N \in \mathbb{N}
$$

implies (2.5). On the other hand, if (2.6) holds, the assumption (H2) for $n$ even is fulfilled. Indeed, for large $A>>1$ and $\operatorname{Im}(\theta) \geq A T$ we have

$$
R_{\psi_{1}, \psi_{2}}(\theta)=-e^{i \theta} \sum_{N=0}^{\infty} \psi_{1} \mathcal{U}(N T, 0) \psi_{2} e^{i N \theta}
$$

and applying (2.6), we conclude that $R_{\psi_{1}, \psi_{2}}(\theta)$ admits a holomorphic extension from $\{\theta \in \mathbb{C}$ : $\operatorname{Im}(\theta) \geq A>0\}$ to $\{\theta \in \mathbb{C}: \operatorname{Im}(\theta)>0\}$. Moreover, $R_{\psi_{1}, \psi_{2}}(\theta)$ is bounded for $\theta \in \mathbb{R}$. We refer to [10] for examples of metrics $a(t, x)$ such that (2.6) is fulfilled. We like to mention that in the study of the time-periodic perturbations of the Schrödinger operators (see [3] ) the resolvent of the monodromy operator $(\mathcal{U}(T)-z)^{-1}$ plays a central role. Moreover, the absence of eigenvalues $z \in \mathbb{C},|z|=1$ of $\mathcal{U}(T)$, and the behavior of the resolvent for $z$ near 1 , are closely related to the decay of local energy as $t \rightarrow \infty$. So our results may be considered as a natural extension of those for Schrödinger operator. On the other hand, for the wave equation we may have poles $\theta \in \mathbb{C}$, $\operatorname{Im} \theta>0$ of the $R_{\psi_{1}, \psi_{2}}(\theta)$, while for the Schrödinger operator with time-periodic potentials such a phenomenon is excluded.

\section{LOCAL TIME EXISTENCE}

In this section we assume $n \geq 3$ and let $a(t, x)$ be a $C^{\infty}$ function on $\mathbb{R}^{n+1}$ satisfying the conditions (1.2). Motivated by the work of T. Tao and M. Keel in [12], we will apply Theorem 1 to find $k>1$ for which the problem (1.1) is locally well-posed. For this purpose we need to find $k>1$ so that there exist $2 \leq p, q<+\infty$ satisfying (2.1) with $\gamma=1$ for which we have

$$
k=\frac{q}{2}, \quad \frac{k}{p}<1 .
$$


Then it is easy to see that $k>1$ satisfies (3.1) with $p, q$ satisfying (2.1), if the following conditions are fulfilled:

$$
\begin{aligned}
& \text { i) } n=3,3<k<5 \\
& \text { ii) } n=4,2<k<3 \\
& \text { iii) } n=5, \frac{5}{3}<k<\frac{7}{3} \\
& \text { iv) } n \geq 6, \frac{n}{n-2}<k \leq \frac{n}{n-3} .
\end{aligned}
$$

Now we recall a version of the Christ-Kiselev lemma.

Lemma 1. Let $X$ and $Y$ be Banach spaces, and for all $s, t \in \mathbb{R}^{+}$let $K(s, t)$ be an operator from $X$ to $Y$. Suppose that

$$
\left\|\int_{0}^{t_{0}} K(s, t) h(s) d s\right\|_{L^{l}\left(\left[t_{0},+\infty[, Y)\right.\right.} \leq A\|h\|_{L^{r}\left(\mathbb{R}^{+}, X\right)},
$$

for some $A>0,1 \leq r<l \leq+\infty$, all $t_{0} \in \mathbb{R}^{+}$and $h \in L^{r}\left(\mathbb{R}^{+}, X\right)$. Then we have

$$
\left\|\int_{0}^{t} K(s, t) h(s) d s\right\|_{L^{l}\left(\mathbb{R}^{+}, Y\right)} \leq A C_{r, l}\|h\|_{L^{r}\left(\mathbb{R}^{+}, X\right)},
$$

where $C_{r, l}>0$ depends only on $r, l$.

We refer to [7] for the proof of Lemma 1 (see also the original paper [1]). Notice that in [7] the above result is formulated with $\mathbb{R}$ instead of $\mathbb{R}^{+}$and $s, t, t_{0} \in \mathbb{R}$, but, as it was mentioned in [7], the same proof works for intervals and in particular for $\mathbb{R}^{+}$. We need the following

Lemma 2. Let $a(t, x)$ satisfy the conditions (1.2). Let $T_{1} \leq \delta$, and $2 \leq p, q<+\infty$ satisfy the conditions (2.1). Then for all $h \in L^{1}\left(\left[0, T_{1}\right], L^{2}\left(\mathbb{R}^{n}\right)\right)$ we have

$$
\left\|\int_{0}^{t} V(t, s) h(s) d s\right\|_{L^{p}\left(\left[0, T_{1}\right], L^{q}\left(\mathbb{R}^{n}\right)\right)} \leq C\|h\|_{L^{1}\left(\left[0, T_{1}\right], L^{2}\left(\mathbb{R}^{n}\right)\right)}
$$

with $C>0$ independent of $T_{1}$.

Proof. Let $t_{0} \in\left[0, T_{1}\right]$. We have

$$
\left\|\int_{0}^{t_{0}} V(t, s) h(s) \mathrm{d} s\right\|_{L^{p}\left(\left[t_{0}, T_{1}\right], L^{q}\left(\mathbb{R}^{n}\right)\right)} \leq \int_{0}^{t_{0}}\|V(t, s) h(s)\|_{L^{p}\left(\left[t_{0}, T_{1}\right], L^{q}\left(\mathbb{R}^{n}\right)\right)} \mathrm{d} s .
$$

From the definition of $V(t, s)$ we know that

$$
\begin{aligned}
\|V(t, s) h(s)\|_{L^{p}\left(\left[t_{0}, T_{1}\right], L^{q}\left(\mathbb{R}^{n}\right)\right)} & =\left\|(\mathcal{U}(t, s)(0, h(s)))_{1}\right\|_{L^{p}\left(\left[t_{0}, T_{1}\right], L^{q}\left(\mathbb{R}^{n}\right)\right)} \\
& \leq \|\left(\mathcal{U}(t, 0)(\mathcal{U}(0, s)(0, h(s)))_{1} \|_{L^{p}\left(\left[0, T_{1}\right], L^{q}\left(\mathbb{R}^{n}\right)\right)} .\right.
\end{aligned}
$$

Then, the estimate (2.2) implies that for all $s \in\left[0, t_{0}\right]$ we obtain

$$
\begin{aligned}
\|\left(\mathcal{U}(t, 0)(\mathcal{U}(0, s)(0, h(s)))_{1} \|_{L^{p}\left(\left[0, T_{1}\right], L^{q}\left(\mathbb{R}^{n}\right)\right)}\right. & \leq C_{\delta}\|\mathcal{U}(0, s)(0, h(s))\|_{\dot{\mathcal{H}}_{1}\left(\mathbb{R}^{n}\right)} \\
& \leq C_{\delta}^{\prime}\|h(s)\|_{L^{2}\left(\mathbb{R}^{n}\right)},
\end{aligned}
$$

where $C_{\delta}^{\prime}=C_{\delta} \sup _{s \in\left[0, T_{1}\right]}\|\mathcal{U}(0, s)\|$ is independent of $t_{0}$. It follows

$$
\begin{aligned}
\left\|\int_{0}^{t_{0}} V(t, s) h(s) \mathrm{d} s\right\|_{L^{p}\left(\left[t_{0}, T_{1}\right], L^{q}\left(\mathbb{R}^{n}\right)\right)} & \leq C_{\delta}^{\prime} \int_{0}^{t_{0}}\|h(s)\|_{L^{2}\left(\mathbb{R}^{n}\right)} \mathrm{d} s \\
& \leq C_{\delta}^{\prime}\|h\|_{L^{1}\left(\left[0, T_{1}\right], L^{2}\left(\mathbb{R}^{n}\right)\right)} .
\end{aligned}
$$


Consider $K(s, t)=\mathbb{1}_{\left[0, T_{1}\right]}(t) \mathbb{1}_{\left[0, T_{1}\right]}(s) V(t, s), X=L^{2}\left(\mathbb{R}^{n}\right)$ and $Y=L^{q}\left(\mathbb{R}^{n}\right)$. Since $p>1$, the Christ-Kiselev lemma yields

$$
\left\|\int_{0}^{t} V(t, s) h(s) \mathrm{d} s\right\|_{L^{p}\left(\left[0, T_{1}\right], L^{q}\left(\mathbb{R}^{n}\right)\right)} \leq C(\delta, p)\|h\|_{L^{1}\left(\left[0, T_{1}\right], L^{2}\left(\mathbb{R}^{n}\right)\right)} .
$$

Applying (3.3), we will show that problem (1.1) is locally well-posed for $k$ and $n$ satisfying the conditions (3.2).

Theorem 3. Assume that $a(t, x)$ is a $C^{\infty}$ function on $\mathbb{R}^{n+1}$ satisfying conditions (1.2) and let $k$ and $n$ satisfy (3.2). Then there exists $T_{1}>0$ such that problem (1.1) admits a weak solution $u$ on $\left[0, T_{1}\right]$. Moreover, $u$ is the unique weak solution of (1.1) on $\left[0, T_{1}\right]$ satisfying the following properties:

$$
\begin{aligned}
& \text { (i) } u \in \mathcal{C}\left(\left[0, T_{1}\right], \dot{H}^{1}\left(\mathbb{R}^{n}\right)\right), \quad(i i) u_{t} \in \mathcal{C}\left(\left[0, T_{1}\right], L^{2}\left(\mathbb{R}^{n}\right)\right), \\
& \text { (iii) } u \in L^{p}\left(\left[0, T_{1}\right], L^{2 k}\left(\mathbb{R}^{n}\right)\right) \quad \text { with } \frac{1}{p}=\frac{n(k-1)}{k}-1 .
\end{aligned}
$$

Proof. Let $k$ and $n$ satisfy (3.2). We have seen that we can find $2 \leq p, q<+\infty$ satisfying conditions (2.1) so that $\frac{k}{p}<1$ and $\frac{k}{q}=\frac{1}{2}$. Consider the norm $\|\cdot\|_{Y_{T_{1}}}$ defined by

$$
\|u\|_{Y_{T 1}}=\|u\|_{\mathcal{C}\left(\left[0, T_{1}\right], \dot{H}^{1}\right)}+\|u\|_{L^{p}\left(\left[0, T_{1}\right], L^{q}\left(\mathbb{R}^{n}\right)\right)}
$$

and

$$
Y_{T_{1}}=\mathcal{C}\left(\left[0, T_{1}\right], \dot{H}^{1}\right) \bigcap L^{p}\left(\left[0, T_{1}\right], L^{q}\left(\mathbb{R}^{n}\right)\right)
$$

with $T_{1}$ to be determined. Notice that $\left(Y_{T_{1}},\|\cdot\|_{Y_{T_{1}}}\right)$ is a Banach space. Assume $f \in \dot{\mathcal{H}}_{1}\left(\mathbb{R}^{n}\right), M>0$ and let $B_{M}=\left\{u \in Y_{T_{1}}:\|u\|_{Y_{T_{1}}} \leq M\right\}$, with $M$ to be determined. The problem of finding a weak solution $u$ of (1.1) is equivalent to find a fixed point of the map

$$
\mathcal{G}(u)=(\mathcal{U}(t, 0) g)_{1}+\int_{0}^{t} V(t, s) f_{k}(u(s)) \mathrm{d} s .
$$

Let $u \in B_{M}$. We have

$$
\begin{aligned}
\left\|\int_{0}^{t} V(t, s) f_{k}(u(s)) \mathrm{d} s\right\|_{\mathcal{C}\left(\left[0, T_{1}\right], \dot{H}^{1}\right)} & \leq \sup _{t \in\left[0, T_{1}\right]} \int_{0}^{T_{1}} \mathbb{1}_{[0, t]}(s)\left\|V(t, s) f_{k}(u(s))\right\|_{\dot{H}^{1}} \mathrm{~d} s \\
& \leq \sup _{t \in\left[0, T_{1}\right]} \int_{0}^{T_{1}}\left\|V(t, s) f_{k}(u(s))\right\|_{\dot{H}^{1}} \mathrm{~d} s .
\end{aligned}
$$

The estimates (2.2) imply that for $T_{1} \leq \delta$ there exists $C>0$ independent of $T_{1}$ such that

$$
\left\|\int_{0}^{t} V(t, s) f_{k}(u(s)) \mathrm{d} s\right\|_{\mathcal{C}\left(\left[0, T_{1}\right], \dot{H}^{1}\right)} \leq C \int_{0}^{T_{1}}\left\|f_{k}(u(s))\right\|_{L^{2}\left(\mathbb{R}^{n}\right)} \mathrm{d} s \leq C_{1} \int_{0}^{T_{1}}\left\||u|^{k}(s)\right\|_{L^{2}\left(\mathbb{R}^{n}\right)} \mathrm{d} s .
$$

On the other hand, Lemma 2 yields

$$
\left\|\int_{0}^{t} V(t, s) f_{k}(u(s)) \mathrm{d} s\right\|_{L^{p}\left(\left[0, T_{1}\right], L^{q}\left(\mathbb{R}^{n}\right)\right)} \leq C_{2} \int_{0}^{T_{1}}\left\||u|^{k}(s)\right\|_{L^{2}\left(\mathbb{R}^{n}\right)} \mathrm{d} s .
$$

We deduce from (3.4) and (3.5) that

$$
\left\|\int_{0}^{t} V(t, s) f_{k}(u(s)) \mathrm{d} s\right\|_{Y_{T_{1}}} \leq C_{3} \int_{0}^{T_{1}}\left\||u|^{k}(s)\right\|_{L^{2}\left(\mathbb{R}^{n}\right)} \mathrm{d} s=C_{3} \int_{0}^{T_{1}}\|u(s)\|_{L^{q}}^{k} \mathrm{~d} s .
$$


Since $\frac{k}{p}<1$, an application of the Hölder inequality yields

$$
\left\|\int_{0}^{t} V(t, s) f_{k}(u(s)) \mathrm{d} s\right\|_{Y_{T_{1}}} \leq C_{3}\|u\|_{L^{p}\left(\left[0, T_{1}\right], L^{q}\left(\mathbb{R}^{n}\right)\right)}^{k}\left(T_{1}\right)^{1-\frac{k}{p}} \leq C_{3} M^{k}\left(T_{1}\right)^{1-\frac{k}{p}} .
$$

Let $M$ be such that $\frac{M}{2} \geq 2 C\left(\left\|g_{1}\right\|_{\dot{H}^{1}}+\left\|g_{2}\right\|_{L^{2}}\right)$ and let $T_{1}$ be small enough such that

$$
C_{3} M^{k}\left(T_{1}\right)^{1-\frac{k}{p}} \leq \frac{M}{2} .
$$

Then $\|\mathcal{G}(u)\|_{Y_{T_{1}}} \leq M$ and $\mathcal{G}(u) \in Y_{T_{1}}$. We have $\mathcal{G}\left(B_{M}\right) \subset B_{M}$ and $B_{M}$ is a closed set of the Banach space $\left(Y_{T_{1}},\|\cdot\|_{Y_{T_{1}}}\right)$. Now we will show that we can choose $T_{1}$ small enough so that $\mathcal{G}$ becomes a contraction. Let $u, v \in B_{M}$. We know that

$$
\mathcal{G}(u)-\mathcal{G}(v)=\int_{0}^{t} V(t, s)\left(f_{k}(u(s))-f_{k}(v(s))\right) \mathrm{d} s .
$$

In the same way as in the proof of inequality (3.4), Theorem 1 and Lemma 2 imply that

$$
\|\mathcal{G}(u)-\mathcal{G}(v)\|_{Y_{T_{1}}} \leq C_{4} \int_{0}^{T_{1}}\left\|f_{k}(u(s))-f_{k}(v(s))\right\|_{L^{2}} \mathrm{~d} s .
$$

On the other hand, $f_{k}$ satisfies

$$
\left|f_{k}(u)-f_{k}(v)\right| \leq C_{5}|u-v|(|u|+|v|)^{k-1}
$$

Consequently,

$$
\|\mathcal{G}(u)-\mathcal{G}(v)\|_{Y_{T_{1}}} \leq C_{6} \int_{0}^{T_{1}}\left\||u(s)-v(s)|(|u(s)|+|v(s)|)^{k-1}\right\|_{L^{2}} \mathrm{~d} s .
$$

Since $\frac{k-1}{q}+\frac{1}{q}=\frac{k}{q}=\frac{1}{2}$, by the generalized Hölder's inequality, we have

$$
\begin{aligned}
\left\||u(s)-v(s)|(|u(s)|+|v(s)|)^{k-1}\right\|_{L^{2}} & \leq\|u-v\|_{L^{q}}\left\|(|u(s)|+|v(s)|)^{k-1}\right\|_{L^{\frac{q}{k-1}}} \\
& \leq\|u-v\|_{L^{q}}\left(\|u\|_{L^{q}}+\|v\|_{L^{q}}\right)^{k-1} .
\end{aligned}
$$

This leads to

$$
\|\mathcal{G}(u)-\mathcal{G}(v)\|_{Y_{T_{1}}} \leq C_{7} \int_{0}^{T_{1}}\|u(s)-v(s)\|_{L^{q}}\left(\|u(s)\|_{L^{q}}+\|v(s)\|_{L^{q}}\right)^{k-1} \mathrm{~d} s .
$$

Applying Hölder's inequality ones more, we find

$$
\|\mathcal{G}(u)-\mathcal{G}(v)\|_{Y_{T_{1}}} \leq C_{7}\left(T_{1}\right)^{1-\frac{k}{p}} 2^{k-1} M^{k-1}\|u-v\|_{L^{p}\left(\left[0, T_{1}\right], L^{q}\left(\mathbb{R}^{n}\right)\right)} \leq C_{7}\left(T_{1}\right)^{1-\frac{k}{p}}(2 M)^{k-1}\|u-v\|_{Y_{T_{1}}} .
$$

Thus, if we choose $T_{1}$ so that

$$
C_{7}(2 M)^{k-1}\left(T_{1}\right)^{1-\frac{k}{p}}<1,
$$

$\mathcal{G}$ will be a contraction from $B_{M}$ to $B_{M}$. Consequently, there exists a unique $u \in Y_{T_{1}}$ such that

$$
\|u\|_{Y_{T_{1}}} \leq M \quad \text { and } \quad \mathcal{G}(u)=u
$$

Remark 2. In contrast to the case $a=1$ (see [12], [13] and [15]) for our argument we must use homogeneous Strichartz estimates. This restriction leads to a solution in the energy space $\dot{\mathcal{H}}_{1}\left(\mathbb{R}^{n}\right)$. Moreover, we have more restrictions on the values of $k>1$. 
Since we use estimates $(2.2)$ to prove Theorem 3 , the length $T_{1}$ of the interval $\left[0, T_{1}\right]$ on which the existence result holds, is majored by the length $\delta$ of the interval on which estimates (2.2) are established. To improve this existence result, in the same way as in [12], we will apply global estimates in the next section.

\section{LONG TIME EXISTENCE FOR SMALL INITIAL DATA}

In this section we assume that $n \geq 3, a(t, x)$ is $T$-periodic with respect to $t$ and (H1), (H2) are fulfilled. We will use the estimates (2.4) to find solutions of (1.1) defined in $\left[0, T_{1}\right]$, with $T_{1}$ only depending on $k, n$ and $g$. For this purpose we must find $k>1$ such that there exist $2 \leq p, q<+\infty$ satisfying (2.3) for which

$$
k=\frac{q}{2}, \quad \frac{k}{p}<1 .
$$

Then $k>1$ satisfies (4.1) with $p, q$ satisfying (2.3) if the following conditions are fulfilled

$$
\begin{aligned}
& \text { i) } n=3,3<k<5 \\
& \text { ii) } n=4,2<k<3 \\
& \text { iii) } n=5, \frac{5}{3}<k<\frac{7}{3}, \\
& \text { iv) } n \geq 6, \frac{n}{n-2}<k<\frac{n}{n-3} .
\end{aligned}
$$

Lemma 3. Assume that (H1) and (H2) are fulfilled, $a(t, x)$ is T-periodic with respect to $t$ and $n \geq 3$. Let $t \geq s \geq 0$. Then

$$
\|\mathcal{U}(t, s)\|_{\mathcal{L}\left(\dot{\mathcal{H}}_{1}\left(\mathbb{R}^{n}\right)\right)} \leq C_{0}
$$

with $C_{0}>0$ independent of $s$ and $t$.

Proof. Let $m \in \mathbb{N}$ be such that $0 \leq s-m T<T$. We have

$$
\mathcal{U}(t, s)=\mathcal{U}(t-m T, s-m T)=\mathcal{U}(t-m T, 0) \mathcal{U}(0, s-m T) .
$$

Since $t-m T \geq s-m T \geq 0$, Theorem 2 implies

$$
\|\mathcal{U}(t-m T, 0)\|_{\mathcal{L}\left(\dot{\mathcal{H}}_{1}\left(\mathbb{R}^{n}\right)\right)} \leq C^{\prime}
$$

with $C^{\prime}>0$ independent of $t$. Also we have

$$
\|\mathcal{U}(0, s-m T)\|_{\mathcal{L}\left(\dot{\mathcal{H}}_{1}\left(\mathbb{R}^{n}\right)\right)} \leq \sup _{s^{\prime} \in[0, T]}\left\|\mathcal{U}\left(0, s^{\prime}\right)\right\|_{\mathcal{L}\left(\dot{\mathcal{H}}_{1}\left(\mathbb{R}^{n}\right)\right)}=C^{\prime \prime}
$$

It follows that

$$
\|\mathcal{U}(t, s)\|_{\mathcal{L}\left(\dot{\mathcal{H}}_{1}\left(\mathbb{R}^{n}\right)\right)} \leq C^{\prime} C^{\prime \prime}=C_{0}
$$

and $C_{0}$ is independent of $t$ and $s$.

The estimates (2.4), the Christ-Kiselev lemma and Lemma 3 imply the following

Lemma 4. Assume $n \geq 3$ and let $a(t, x)$ be T-periodic with respect to $t$ such that (H1) and (H2) are fulfilled. Let $2 \leq p, q<+\infty$ satisfy condition (2.3) and let $T_{1}>0$. Then for all $h \in L^{1}\left(\left[0, T_{1}\right], L^{2}\left(\mathbb{R}^{n}\right)\right)$ we have

$$
\left\|\int_{0}^{t} V(t, s) h(s) d s\right\|_{L^{p}\left(\left[0, T_{1}\right], L^{q}\left(\mathbb{R}^{n}\right)\right)} \leq C\|h\|_{L^{1}\left(\left[0, T_{1}\right], L^{2}\left(\mathbb{R}^{n}\right)\right)}
$$

with $C>0$ independent of $g$ and $T_{1}$. 
Proof. Let $t_{0}>0, s \in\left[0, t_{0}\right]$ and $t>t_{0}$. Consider $m T \leq t_{0}<(m+1) T$. We have

$$
\begin{aligned}
V(t, s) h(s) & =(\mathcal{U}(t-m T, s-m T)(0, h(s)))_{1} \\
& =(\mathcal{U}(t-m T, 0) \mathcal{U}(0, s-m T)(0, h(s)))_{1} \\
& =(\mathcal{U}(t-m T, 0) \mathcal{U}(m T, s)(0, h(s)))_{1} .
\end{aligned}
$$

Thus, the estimate (2.4) implies

$$
\left\|\int_{0}^{t_{0}} V(t, s) h(s) \mathrm{d} s\right\|_{L^{p}\left(\left[t_{0},+\infty\left[, L^{q}\left(\mathbb{R}^{n}\right)\right)\right.\right.} \leq C \int_{0}^{t_{0}}\|\mathcal{U}(m T, s)(0, h(s))\|_{\dot{\mathcal{H}}_{1}\left(\mathbb{R}^{n}\right)} \mathrm{d} s .
$$

Since $0 \leq s \leq m T$ for $s \in[0, m T]$, Lemma 3 yields

$$
\int_{0}^{m T}\|\mathcal{U}(m T, s)(0, h(s))\|_{\dot{\mathcal{H}}_{1}\left(\mathbb{R}^{n}\right)} \mathrm{d} s \leq C_{0} \int_{0}^{m T}\|h(s)\|_{L^{2}\left(\mathbb{R}^{n}\right)} \mathrm{d} s
$$

with $C>0$ independent of $t_{0}$. In the same way, since $m T \leq t_{0}<(m+1) T$ we have

$$
\int_{m T}^{t_{0}}\|\mathcal{U}(0, s-m T)(0, h(s))\|_{\dot{\mathcal{H}}_{1}\left(\mathbb{R}^{n}\right)} \mathrm{d} s \leq \sup _{s \in[0, T]}\|\mathcal{U}(0, s)\| \int_{m T}^{t_{0}}\|h(s)\|_{L^{2}\left(\mathbb{R}^{n}\right)} \mathrm{d} s .
$$

It follows that

$$
\left\|\int_{0}^{t_{0}} V(t, s) h(s) \mathrm{d} s\right\|_{L^{p}\left(\left[t_{0},+\infty\left[, L^{q}\left(\mathbb{R}^{n}\right)\right)\right.\right.} \leq C \int_{0}^{+\infty}\|h(s)\|_{L^{2}\left(\mathbb{R}^{n}\right)} \mathrm{d} s .
$$

Since $p>1$, the Christ- Kiselev lemma implies

$$
\left\|\int_{0}^{t} V(t, s) h(s) \mathrm{d} s\right\|_{L^{p}\left(\mathbb{R}^{+}, L^{q}\left(\mathbb{R}^{n}\right)\right)} \leq C_{p}\|h\|_{L^{1}\left(\mathbb{R}^{+}, L^{2}\left(\mathbb{R}^{n}\right)\right)}
$$

We deduce that

$$
\begin{aligned}
\left\|\int_{0}^{t} V(t, s) h(s) \mathrm{d} s\right\|_{L^{p}\left(\left[0, T_{1}\right], L^{q}\left(\mathbb{R}^{n}\right)\right)} & \leq\left\|\int_{0}^{t} V(t, s) \mathbb{1}_{\left[0, T_{1}\right]}(s) h(s) \mathrm{d} s\right\|_{L^{p}\left(\mathbb{R}^{+}, L^{q}\left(\mathbb{R}^{n}\right)\right)} \\
& \leq C\|h\|_{L^{1}\left(\left[0, T_{1}\right], L^{2}\left(\mathbb{R}^{n}\right)\right)}
\end{aligned}
$$

with $C>0$ independent of $T_{1}$.

Theorem 4. Assume that $k$ and $n$ satisfy the conditions (4.2). Let $a(t, x)$ be T-periodic with respect to $t$ and let (H1), (H2) be fulfilled. Then there exists $C\left(k, f_{k}, T, \rho, n\right)$ such that for all $g \in \dot{\mathcal{H}}_{1}\left(\mathbb{R}^{n}\right)$ we can find a weak solution $u$ of (1.1) on $\left[0, T_{1}\right]$ with

$$
T_{1}=C\left(k, f_{k}\right)\left(\|g\|_{\dot{\mathcal{H}}_{1}\left(\mathbb{R}^{n}\right)}\right)^{-d}
$$

where $d=\frac{2(k-1)}{(n+2)-(n-2) k}$. Moreover, $u$ is the unique weak solution of (1.1) on $\left[0, T_{1}\right]$ satisfying the following properties:

$$
\begin{aligned}
& \text { (i) } u \in \mathcal{C}\left(\left[0, T_{1}\right], \dot{H}^{1}\left(\mathbb{R}^{n}\right)\right), \quad(i i) u_{t} \in \mathcal{C}\left(\left[0, T_{1}\right], L^{2}\left(\mathbb{R}^{n}\right)\right), \\
& \text { (iii) } u \in L^{p}\left(\left[0, T_{1}\right], L^{2 k}\left(\mathbb{R}^{n}\right)\right) \quad \text { with } \quad \frac{1}{p}=\frac{n(k-1)}{k}-1 .
\end{aligned}
$$

Proof. Let $C_{f}>0$ be such that $\left|f_{k}(u)\right| \leq C_{f}|u|^{k}$ and $\left|f_{k}(u)-f_{k}(v)\right| \leq C_{f}|u-v|(|u|+|v|)^{k-1}$. Then, Theorem 2 and Lemma 4 imply that there exists $A_{k}$ such that for all $T_{1}>0$

$$
\left\|\int_{0}^{t} V(t, s) h(s) \mathrm{d} s\right\|_{L^{p}\left(\left[0, T_{1}\right], L^{q}\left(\mathbb{R}^{n}\right)\right)} \leq A_{k}\|h\|_{L^{1}\left(\left[0, T_{1}\right], L^{2}\left(\mathbb{R}^{n}\right)\right)}
$$


and

$$
\left\|(\mathcal{U}(t, 0) g)_{1}\right\|_{L^{p}\left(\left[0, T_{1}\right], L^{q}\left(\mathbb{R}^{n}\right)\right)} \leq A_{k}\|g\|_{\dot{\mathcal{H}}_{1}\left(\mathbb{R}^{n}\right)} .
$$

According to the proof of Theorem $3, \mathcal{G}(u)=(\mathcal{U}(t, 0) g)_{1}+\int_{0}^{t} V(t, s) f_{k}(u(s)) \mathrm{d} s$ admits a fixed point in the set

$$
\left\{u \in \mathcal{C}\left(\left[0, T_{1}\right], \dot{H}^{1}\right) \cap L^{p}\left(\left[0, T_{1}\right], L^{q}\right):\|u\|_{\mathcal{C}\left(\left[0, T_{1}\right], \dot{H}^{1}\right)}+\|u\|_{L^{p}\left(\left[0, T_{1}\right], L^{q}\right)} \leq M\right\}
$$

if we choose $M, T_{1}>0$ so that

$$
\left\{\begin{array}{l}
A_{k}\|g\|_{\dot{\mathcal{H}}_{1}\left(\mathbb{R}^{n}\right)}+C_{3} M^{k}\left(T_{1}\right)^{1-\frac{k}{p}} \leq M, \\
C_{7}(2 M)^{k-1}\left(T_{1}\right)^{1-\frac{k}{p}}<1 .
\end{array}\right.
$$

In particular (4.5) will be fulfilled if

$$
\left\{\begin{array}{l}
A_{k}\|g\|_{\dot{\mathcal{H}}_{1}\left(\mathbb{R}^{n}\right)}+C_{3} M^{k}\left(T_{1}\right)^{1-\frac{k}{p}}=M, \\
C_{7}(2 M)^{k-1}\left(T_{1}\right)^{1-\frac{k}{p}}<1 .
\end{array}\right.
$$

We will choose $M, T_{1}$ so that (4.6) holds. Let $t_{1}=\left(T_{1}\right)^{1-\frac{k}{p}}$. We find that the system (4.6) is equivalent to the following

$$
\left\{\begin{array}{l}
t_{1}=\frac{M-A_{k}\|g\|_{\dot{\mathcal{H}}_{1}\left(\mathbb{R}^{n}\right)}}{C_{3} M^{k}}, \\
0<\frac{M-A_{k}\|g\|_{\dot{\mathcal{H}}_{1}\left(\mathbb{R}^{n}\right)}}{M}<\frac{1}{C_{7} 2^{k-1}} .
\end{array}\right.
$$

Since $M \longmapsto \frac{M-A_{k}\|g\|_{\dot{\mathcal{H}}_{1}\left(\mathbb{R}^{n}\right)}}{M}$ is strictly increasing, we obtain that $\left(t_{1}, M\right)$ is a solution of $(4.7)$ if

$$
M<\frac{2^{k-1} A_{k}\|g\|_{\dot{\mathcal{H}}_{1}\left(\mathbb{R}^{n}\right)}}{2^{k-1}-1} .
$$

Take

$$
M_{0}=\frac{\alpha 2^{k-1} A_{k}\|g\|_{\dot{\mathcal{H}}_{1}\left(\mathbb{R}^{n}\right)}}{2^{k-1}-1} \quad \text { and } \quad t_{1}=\frac{M_{0}-A_{k}\|g\|_{\dot{\mathcal{H}}_{1}\left(\mathbb{R}^{n}\right)}}{C_{3}\left(M_{0}\right)^{k}}
$$

with $1-\frac{1}{2^{k-1}}<\alpha<1$. Then $\left(M_{0}, t_{1}\right)$ is a solution of $(4.7)$ and we have

$$
t_{1}=\frac{\frac{\alpha k^{k-1}}{2^{k-1}-1}-1}{C_{8}\left(\frac{2^{k-2} A_{k}}{2^{k-1}-1}\right)^{k}\|g\|_{\dot{\mathcal{H}}_{1}\left(\mathbb{R}^{n}\right)}^{k-1}}=C^{\prime}\left(k, f_{k}\right)\|g\|_{\dot{\mathcal{H}}_{1}\left(\mathbb{R}^{n}\right)}^{-(k-1)} .
$$

Thus for $M=M_{0}$ and

$$
T_{1}=\left(t_{1}\right)^{\frac{1}{1-\frac{k}{p}}}=C\left(k, f_{k}\right)\left(\|g\|_{\dot{\mathcal{H}}_{1}\left(\mathbb{R}^{n}\right)}\right)^{-\frac{k-1}{1-\frac{k}{p}}}
$$

$M$ and $T_{1}$ satisfy conditions (4.6). Moreover, we know that

$$
\frac{k}{p}=\frac{(n-2) k-n}{2} \text {. }
$$


Thus we have

$$
\frac{k-1}{1-\frac{k}{p}}=\frac{2(k-1)}{(n+2)-(n-2) k}
$$

and $M, T_{1}$ satisfy conditions (4.5) if $M=M_{0}$ and

$$
T_{1}=C\left(k, f_{k}\right)\left(\|g\|_{\dot{\mathcal{H}}_{1}\left(\mathbb{R}^{n}\right)}\right)^{-\frac{2(k-1)}{(n+2)-(n-2) k}} .
$$

Note that for $n \geq 6$ we have $\frac{n}{n-3} \leq \frac{n+2}{n-2}$ and $k<\frac{n}{n-3}$ leads to $k<\frac{n+2}{n-2}$.

Remark 3. Let $\|g\|_{\dot{\mathcal{H}}_{1}\left(\mathbb{R}^{n}\right)}=\epsilon$ and $T_{1}=C \epsilon^{-d}, C, d>0$ being the constants defined by (4.3). Then Theorem 4 implies that there exists a unique solution of (1.1) satisfying (4.4).

\section{REFERENCES}

[1] M. Christ and A. Kiselev, Maximal functions associated to filtrations, J. Funct. Anal., 179(2) (2001), 409-425.

[2] F. Colombini and J. Rauch, Smooth Localised Parametric Resonance for Wave Equations, J. Reine Angew. Math., 616 (2008), 1-14.

[3] A. Galtabayar, A. Yensen and K. Yajima, Local time-decay of solutions to Schrödinger equations with timeperiodic potentials, Journal of Statistical Physics, 114 (2004), 231-281.

[4] M. G. Grillakis, Regularity and asymptotic behavior of the wave equation with a critical non-linearity, Ann. of Math., 132 (1990), 485-509.

[5] J. Ginibre and G. Velo Scattering theory in the energy space for a class of nonlinear wave equations, Comm. Math. Phys., 123 (1989), 535-573.

[6] J. Ginibre and G. Velo Regularity of solutions of critical and subcritical nonlinear wave equations, Nonlinear Anal., 22 (1994), 1-19.

[7] A. Hassell, T. Tao and J. Wunsch, Sharp Strichartz Estimates on Non-trapping Asymptotically Conic Manifols, Amer. J. Math., 128 (2006), no 4, 963-1024.

[8] L. Kapitanski, Weak and yet weaker solutions of semi-linear wave equations, Comm. Partial Differential Equation, 19 (1994), 1629-1676.

[9] Y. Kian, Strichartz estimates for the wave equation with a time-periodic non-trapping metric, Asymptotic Analysis, to appear.

[10] Y. Kian, Local energy decay in even dimensions for the wave equation with a time-periodic non-trapping metric and applications to Strichartz estimates, Serdica Math. J., 36 (2010), 329-370.

[11] S. Klainerman, Global existence for nonlinear wave equations, Comm. on Pure appl. Math., 33 (1980), $43-101$.

[12] M. Keel and T.Tao, Endpoint Strichartz Estimates, Amer. J. Math., 120 (1998), 955-980.

[13] H. Lindelbag and C. D. Sogge, On existence an scattering with minimal regularity for the semilinear wave equation, J. Funct. Anal., 130 (1995), 357-426.

[14] C. Miao and B. Zhang, $H^{s}$-global well-posedness for semilinear wave equations, J. Math. Anal. Appl, 283 (2003), 645-666.

[15] H. Pecher, Nonlinear small data scattering for the wave and Klein-Gordon equation, Math. Z, 185 (1984), 261270.

[16] V. Petkov, Scattering Theory for Hyperbolic Operators, North Holland, Amsterdam, 1989.

[17] V. Petkov, Global Strichartz Estimates for the Wave Equation with Time-periodic Potentials, J. Funct. Anal., 235 (2006), 357-376.

[18] M. Reissig and K. Yagdijian, $L_{p}-L_{q}$ estimates for the solution of strictly hyperbolic equation of second order with increasing in time coefficient, Math. Nachr., 214 (2000), 71-104.

[19] C. D. Sogge, Lectures on nonlinear wave-equations, International Press, Boston, 1995.

[20] R. Strichartz, A priori estimates for the wave equation and some applications, Journal. Funct. Anal, 5 (1970), 218-235. 
Université Bordeaux I, Institut de Mathématiques de Bordeaux, 351, Cours de la Libération, 33405 TAlence, France

E-mail address: Yavar.Kian@math.u-bordeaux1.fr 